\title{
Protective effects and mechanism of TPX2 on neurocyte apoptosis of rats in Alzheimer's disease model
}

\author{
KESHAN LIANG $^{1,2}$, JINGLING ZHANG ${ }^{3}$, CHENGBIN YIN $^{4}$, XUEYING ZHOU ${ }^{5}$ and SHENGNIAN ZHOU ${ }^{1}$ \\ ${ }^{1}$ Department of Neurology, Qilu Hospital of Shandong University and Brain Science Research Institute, Shandong University, \\ Jinan, Shandong 250012; ${ }^{2}$ Department of Neurology, Pingyi Branch of Qilu Hospital, Shandong University, Pingyi, \\ Shandong 273300; ${ }^{3}$ Department of Endocrinology, Linyi People's Hospital, Linyi, Shandong 276000; ${ }^{4}$ Department of \\ Emergency, Qingdao Branch of Qilu Hospital of Shandong University, Qingdao, Shandong 266000; ${ }^{5}$ Department of \\ Neurology, Shangdong University of Traditional Chinese Medicine, Jinan, Shandong 250031, P.R. China
}

Received July 11, 2016; Accepted November 3, 2016

DOI: 10.3892/etm.2016.4006

\begin{abstract}
We investigated the protective effects and mechanism of TPX2 on apoptosis of rat neurocytes. A total of 90 SD rats were randomly divided into the drug group, the control group and the blank group, with 30 rats in each group. The rats in the drug group and in the blank group were anesthetized with $10 \%$ chloral hydrate (at the dose of $0.5 \mathrm{ml} / 100 \mathrm{~g}$ ) and $\mathrm{A} \beta_{1-42}$, with the concentration of $5 \mu \mathrm{l}(1 \mu \mathrm{g} / \mu \mathrm{l})$, was injected in the exact position of bilateral hippocampal areas of rats to establish the model. The configured TPX2 inhibitors and edible benne oil were mixed and made into a suspension. After model establishment, the rats were given different treatment methods; the rats in the drug group were given gavage administration in the proportion of $75 \mathrm{mg} / \mathrm{kg}$ once a day. The rats in the control group were given intragastric administration with the same proportion of physiological saline once a day. The blank group was the normal healthy group and the rats in this group did not undergo any surgery or drug treatment. Brain tissue in rats were divided into two parts, one part was fixed, dehydrated, paraffin-embedded and made into slices of approximately $5 \mu \mathrm{m}$. TUNEL staining was used to examine the apoptosis of brain tissue, H\&E staining was used to observe the brain tissue cells of each group, and western blotting for detecting the MAPK, Erk and expression levels of p38 and RT-polymerase chain reaction method was employed to examine mRNA expression levels of MAPK, Erk and p21. After one week, TUNEL staining showed that apoptosis of brain tissue in the drug group was significantly greater than those of the control and blank groups. The protein expression levels of MAPK, Erk and p38 were significantly higher than those of the control
\end{abstract}

Correspondence to: Dr Shengnian Zhou, Department of Neurology, Qilu Hospital of Shandong University and Brain Science Research Institute, Shandong University, 107 Wenhuaxi Road, Jinan, Shandong 250012, P.R. China

E-mail: zhou_shengnian1@163.com

Key words: Alzheimer's disease, apoptosis, TPX2, MAPK group and the normal healthy group; the differences were statistically significant $(\mathrm{P}<0.05)$. Western blotting showed that the protein expression levels of MAPK, Erk and p38 of the drug group were significantly lower than those of the control group but higher than those of the normal healthy group; the differences were statistically significant $(\mathrm{P}<0.05)$. TPX2 has a protective effect on the apoptosis of brain tissue processed by $\mathrm{A} \beta_{1-42}$, which plays its role through the inhibition of the protein expression levels of MAPK, Erk and p38.

\section{Introduction}

Alzheimer's disease (AD) is a neurodegenerative disease, with the clinical symptoms of cognitive impairment and memory decline. WHO has estimated that by the year 2050 , AD patients in Europe and the United States may reach 30 million (1). There are approximately 6 million AD patients in China, accounting for a third of global AD patients. The number of patients each year has been on the increase at a rate of 1,800,000 individuals annually, which makes the situation very grim. After cardiovascular disease, stroke and cancer, $\mathrm{AD}$ has become the fourth most frequent disease that threatens the life and health of the elderly. Currently, it is believed that the main pathogenesis of AD includes oxidative stress, $\beta$-amyloid cascade reaction, immune inflammation reaction, gene mutation and apoptosis of cerebral tissue $(2,3)$.

Xklp2 target protein (targeting protein for xklp2, TPX2) is a new spindle component of a family of vertebrates, and is a type of nuclear proliferation related protein regulated strictly by the cell cycle. TPX2 plays an important role in the stability of the spindle during mitosis $(4,5)$. The detection of TPX2 levels in cancer tissues is helpful in order to know the status of cell proliferation and apoptosis. Some studies have shown that in the tumor cells, TPX2 overexpression is associated with tumor metastasis and recurrence in various types of cancer, including pancreatic cancer (6), ovarian cancer (7), salivary gland carcinoma (8), colorectal cancer (9) and esophageal carcinoma (10). Currently, the role of TPX2 in the neuronal cell apoptosis of AD and its significance are not clear.

Therefore, the effect and mechanism of TPX2 on nerve cells in AD disease has been relatively limited. The aim of the 
study was to analyze the effects and mechanisms of TPX2 on the apoptosis of neural cells in an AD model.

\section{Materials and methods}

Experimental materials. Taq Master Mix (SinoBio, Walpole, MA, USA), agarose sterile saline, sterile double distilled water, rabbitmonoclonal anti-phospho-MAPK (dilution: 1:1,000; cat no.: 4370; Cell Signaling Technology, Inc., Danvers, MA, USA) and rabbit monoclonal $\beta$-actin antibody (dilution: 1:5,000; cat no.: RB-9421-P; Invitrogen Life Technologies, Carlsbad, CA, USA) and rabbit polyclonal anti-MAPK antibody (dilution: 1:1,000; cat no.: 4695; Cell Signaling Technology, Inc., Danvers, MA, USA), $0.9 \%$ sterile saline (Otsuka pharmaceutical Co., Ltd., Tokyo, Japan), TRIzol (Invitrogen Life Technologies) and pathological slice machine (Leica, Mannheim, Germany).

Experimental equipment. Equipment used for the polymerase chain reaction (PCR) included amplification instrument (Bio-Rad, Berkeley, CA, USA), gel imager (Bio-Rad), electrophoresis apparatus (Beijing Liuyi Instrument Factory, Beijing, China), centrifuge (Eppendorf AG, Hamburg, Germany), trace pipettes (Eppendorf $A G)$, and a Haier ice making machine (Haier Company, Shanghai, China). Equipment used for western blotting included western blotting electrophoresis apparatus (Bio-Rad), $-80^{\circ} \mathrm{C}$ refrigerator (Thermo Fisher Scientific, Waltham, MA, USA), $10 \mathrm{ml}$ syringe, $5 \mathrm{ml}$ syringe (Tianjin Hanaco Medical Co., Ltd., Tianjin, China) and for the animal experiments special surgical instruments (Beijing Medical Instrument Factory, Beijing, China), NanoDrop 2000 spectrophotometer (Thermo Fisher Scientific), Eppendorf tube (Eppendorf AG), and water bath (Beijing Medical Instruments Factory).

Methods. A total of $90 \mathrm{SD}$ rats were randomly divided into the drug group, the control group and the blank group, with 30 rats in each group. The rats in the drug group and the control group rats were anesthetized with $10 \%$ chloral hydrate (at a dose of $0.5 \mathrm{ml} / 100 \mathrm{~g}$ ). After successful anesthesia and the accurate positioning of bilateral hippocampal area of the rats, $\mathrm{A} \beta_{1-42}$, with the concentration of $5 \mu \mathrm{l}(1 \mu \mathrm{g} / \mu \mathrm{l})$, was injected to establish the models. TPX2 inhibitors and edible benne oil were mixed in the proportion of $0.1 \mathrm{~g} / 12.5 \mathrm{ml}$ to make a suspension. After the model was established, the rats were given different treatments. The rats in the drug group were given intragastric administration once a day. The control group was given intragastric administration with the same proportion of physiological saline once a day. The blank group was the normal healthy group, the rats in this group did not undergo any surgery or drug treatment. Three groups of rats were fed for 30 days, and after 30 days, Y-type electric maze was used to detect learning and memory ability of all rats. After the rats were euthanized, all three groups were perfused with PBS until outflow of PBS was bloodless. Brain tissue in rats were divided into two parts, one part was fixed, dehydrated, paraffin-embedded and sectioned (approximately $5 \mu \mathrm{m}$ ). The other part was placed in liquid nitrogen and then preserved at $-80^{\circ} \mathrm{C}$ for detection. TUNEL staining was used to examine the apoptosis of brain tissue under a
Table I. Comparisons of the fluorescence intensity of MAPK and mRNA p38 in the brain tissues of rats in the three groups (mean \pm standard deviation).

\begin{tabular}{lcccc}
\hline Groups & Quantity & TPX2 & MAPK & P38 \\
\hline Drug & 30 & $12.3 \pm 2.4$ & $102.7 \pm 12.6$ & $140.2 \pm 8.9$ \\
Control & 30 & $43.2 \pm 2.4$ & $75.3 \pm 7.9$ & $38.7 \pm 4.6$ \\
Healthy & 30 & $5.28 \pm 1.5$ & $4.37 \pm 2.5$ & $35.7 \pm 2.6$ \\
F-value & - & 10.29 & 12.58 & 30.44 \\
P-value & - & 0.021 & 0.011 & 0.008 \\
\hline
\end{tabular}

microscope (Olympus, Tokyo, Japan). H\&E staining was used to observe the brain tissue cells of each group, western blotting was used to detect the protein expression levels of MAPK, Erk as well as p38, and RT-PCR was employed to examine mRNA expression levels of MAPK, Erk and p21 (11).

RNA: Preparation and reverse transcription (RT)-PCR analysis. The reagents and consumables were all administed without RNA enzyme. The extraction of total RNA was conducted using TRIzol $^{\text {TM }}$ (Invitrogen Life Technologies), and was operated according to the reagent extraction instructions. The extracted RNA was dissolved in DEPC water, and was saved at the temperature of $-80^{\circ} \mathrm{C}$. RT-PCR primers were designed using oligo 6.0 software. The sequence of the TPX2 primers upstream was 5'-ACCTTGCCCTACTAAGATT-3' and TPX2 downstream was 5'-AATGTGGCACAGGTTG AGC-3' prepared by Shanghai Biological Engineering Technology Co). PCR products underwent electrophoresis separation with $1.5 \%$ agarose gel, and SmartView analysis software of FR-980 image analysis system (Shanghai Fudan Biological Electrophoresis Technology Co., Ltd., Shanghai, China) was employed to make density measurement on the products bands. The housekeeping gene GADPH of the same samples was considered as an internal control used to evaluate the transcription levels of $T P X 2$ gene.

Statistical analysis. SPSS 19.0 software (SPSS, Inc., Chicago, IL, USA) was used for data analysis. Quantitative data are presented as mean \pm standard deviation. Single factor combined with LSD method was employed for comparisons among groups. $\mathrm{P}<0.05$ was considered to indicate a statistically significant difference.

\section{Results}

TPX2 protection of apoptosis of brain tissue in rats. We discovered, through the inverted microscope using the TUNEL staining method, that the apoptosis levels of the brain tissue in the drug group rats were significantly increased, and the differences between the drug group and the control group were statistically significant $\mathrm{P}<0.01$ (Fig. $1 \mathrm{~A}$ and $\mathrm{B}$ ).

Inhibition of TPX2 to MAPK, p38 mRNA expression levels. In order to explore the signaling pathway of TPX2, the expression levels of MAPK, p21 mRNA were detected. The results showed that MAPK signaling pathway expression levels of the 

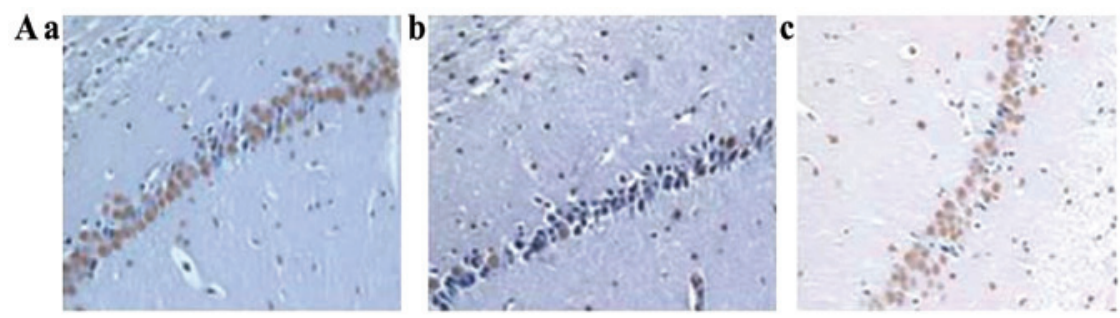

B

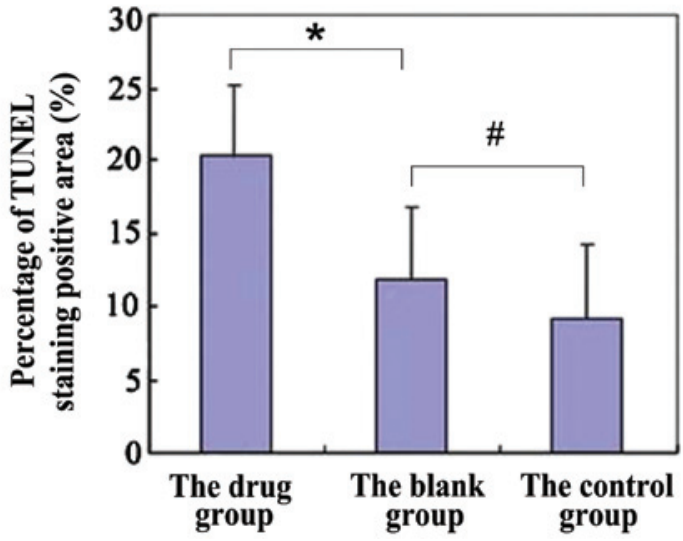

Figure 1. (A) Magnification, $\mathrm{x} 400$ rat brain hippocampus $\mathrm{C} 1$ zone neuron TUNEL staining results. a, drug group; b, blank group; and c, control group. In the blank group, a small amount of TUNEL-positive cell expression is evident. (B) TUNEL-positive cell expression of the blank group increased significantly compared with that of the control group $\left({ }^{\#} \mathrm{P}<0.01\right)$, and TUNEL-positive cell expression of the drug group increased significantly compared with that of the blank group ( $\mathrm{P}<0.01)$.

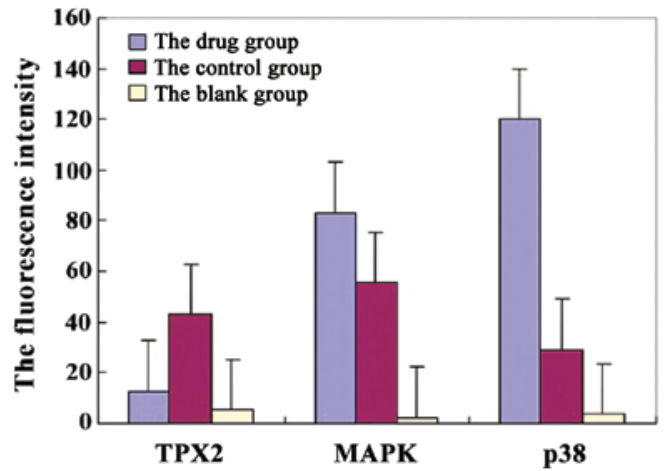

Figure 2. After using TPX2 inhibitor, TPX2 expression levels of the drug group were significantly lower than those of the control group $(\mathrm{P}<0.05)$. MAPK and fluorescence intensity of p21 were detected and compared. The MAPK levels of the drug group improved significantly compared to the normal healthy group, apoptosis of nerve cells increased through the MAPK-p38 signaling pathway.

brain tissue in the drug group rats improved greatly $(\mathrm{P}<0.05)$ and the level of p38 mRNA also increased significantly $(\mathrm{P}<0.05$; Table I, Fig. 2).

Inhibition of TPX2 to MAPK, and p38 mRNA protein expression levels. The MAPK and $\mathrm{p} 38$ protein expression levels of rats in the three groups were detected and compared to each other. We found that $\mathrm{p} 38$ protein expression levels of the three groups had no significant difference $(\mathrm{P}>0.05)$. MAPK levels of the rats were significantly higher than those of rats in the blank group. MAPK levels of rats in the drug groups was significantly lower than those of rats in the control group; differences were statistically significant $(\mathrm{P}<0.05$; Fig. 3$)$.
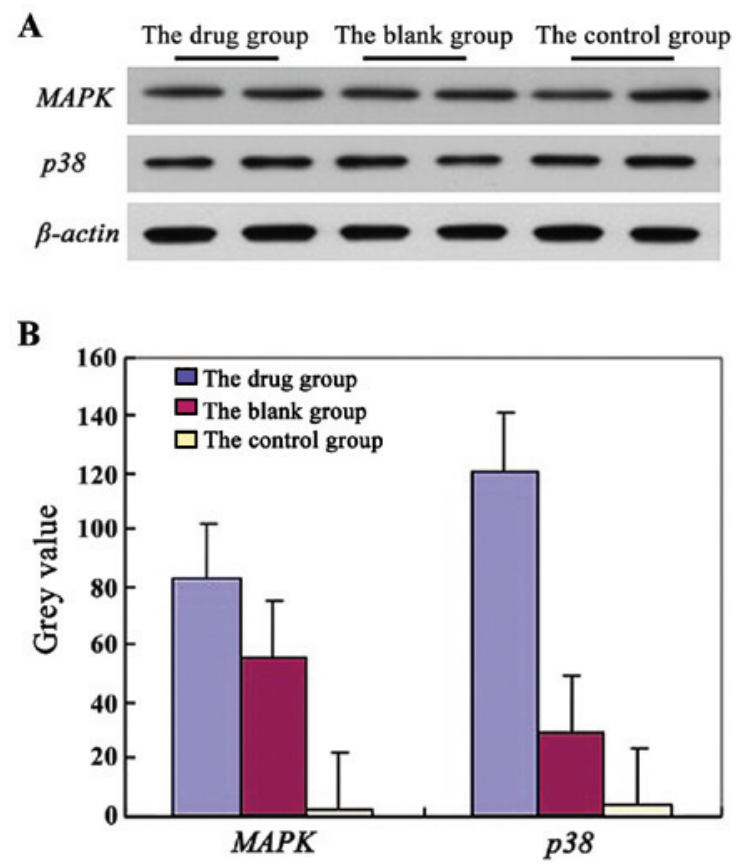

Figure 3. (A) MAPK, and p38 signaling pathway expression levels of the rats in the three groups. (B) It was found from the comparison of western blotting gray values that Erk and p21 levels had no significant difference $(\mathrm{P}>0.05)$ while in the control group, MAPK gray values were significantly higher than those in blank group, and gray values of the drug group decreased significantly. The differences were statistically significant $(\mathrm{P}<0.05)$.

\section{Discussion}

In May 2014, it was proposed by the Alzheimer's Association that exploring $\mathrm{AD}$ biomarkers can increase our understanding of the pathogenesis of the disease, and accelerate 
the development of therapeutic drugs (12). The existing AD biomarkers have shown good application value in clinical diagnosis. Currently, the main pathogenesis of AD involves gene mutation, hyperphosphorylation of the tau protein, oxidative stress, and neuronal apoptosis. Studies have found that the incidence of AD is closely correlated with the presence of $\beta$-amyloid precursor protein gene, presenilin (PS)-1, PS-2 gene and apolipoprotein E gene $(13,14)$. In addition, the tau protein phosphorylation balance is damaged, which further exacerbates the damage of neuronal axon stability and causes functional degradation of the nerve fibers (15). Current studies are mostly confined in molecular biology or tissues, and studies of the role of nerve cells in the pathogenesis of AD and its mechanism are still limited.

TPX2 belongs to the AURKA axis, and as a protein stabilizing the mitotic spindle, which has been previously found in tumors, is clearly related to the malignant proliferation and apoptosis of tumor cells. Through the inhibition of the expression of the TPX2 gene, it can effectively improve tumor cell amplification and migration ability (16). The human TPX2 gene is located on chromosome 20q11.2; its transcription and translation are strictly controlled by the cell cycle, and it is generally expressed in the G-S junctional stage of the cell cycle. TPX2 expression level gradually decreases after the completion of cytokinesis. In the S and G2 phases, the human TPX2 protein usually distributes in the cell nucleus (17). The mitotic stage is closely connected with the spindle, which allows the dynein targeting protein Xklp2 to attach to spindle microtubules. Through the maintenance of normal spindle polarity and analysis of related gene expression profile of exogenous overexpression of DUSP6 in pancreatic cancer cells, Furukawa and colleagues found that AURKA/STK15 gene encoding the Aurora-A kinase gene plays an important role in cell division and leads to a decrease in gene expression of AURKB, TPX2 and CENPA (18). Therefore, the correlation between MAPK activity and AURKA expression, as well as promoter activity, can be proven. The knockout of ETS2 can lead to the downregulation of AURKA expression. These results suggest that in pancreatic cancer, AURKA is a direct target in the MAPK signaling pathway, and at least under the induction of ETS2, AURKA expression levels may increase in pancreatic cancer.

It can be concluded from our study that using TPX2 inhibitors can lead to a significant reduction of nerve tissue apoptosis in rats; differences were statistically significant compared to the control group $(\mathrm{P}<0.05)$. TUNEL staining results showed that apoptotic cells were significantly decreased $(\mathrm{P}<0.05)$. Furthermore, through the detection of the protein expression levels of MAPK, Erk and p38, we found that the proteins in the brain tissues of the rats in the drug group were significantly lower than those of the control group, but higher than those of the blank group; differences were statistically significant $(\mathrm{P}<0.05)$. mRNA levels of MAPK and p38 in the rats of the drug group were higher than those of the control group; differences were statistically significant $(\mathrm{P}<0.05)$. Therefore, we believe that the pro-apoptotic effect of TPX2 inhibitors may play a role through the inhibition of the MAPK-p38 signaling pathway.

In addition, MAPK can regulate endoplasmic reticulum stress and lead to the apoptosis of hepatic stellate cells. In lung cancer, the promotion of the expression of p38-MAPK signaling pathway can promote apoptosis of lung cancer cells, and therefore achieve the purpose of treatment (19-21). In this study, it has been once more proven that MAPK-p38 signaling pathway can play a role in apoptosis of brain tissue in rats. However, this conclusion still needs further supporting research data, such as the use of MAPK inhibitors in the control group. In addition, studies need to be conducted regarding the mechanism behind its protective effect which may further show that MAPK is the main signaling pathway of brain tissue apoptosis.

In conclusion, the results showed that TPX2 has a protective effect on apoptosis of brain tissue processed by $\mathrm{A} \beta_{1-42}$, through the inhibition of the protein expression levels of MAPK, Erk and p38.

\section{References}

1. Lara E, Haro JM, Tang MX, Manly J and Stern Y: Exploring the excess mortality due to depressive symptoms in a community-based sample: The role of Alzheimer's disease. J Affect Disord 202: 163-170, 2016.

2. Steketee RM, Meijboom R, de Groot M, Bron EE, Niessen WJ, van der Lugt A, van Swieten JC and Smits M: Concurrent white and gray matter degeneration of disease-specific networks in early-stage Alzheimer's disease and behavioral variant frontotemporal dementia. Neurobiol Aging 43: 119-128, 2016.

3. Engels MM, Hillebrand A, van der Flier WM, Stam CJ, Scheltens P and van Straaten EC: Slowing of hippocampal activity correlates with cognitive decline in early onset Alzheimer's disease. An MEG study with virtual electrodes. Front Hum Neurosci 10: 238, 2016.

4. Vos JW, Pieuchot L, Evrard JL, Janski N, Bergdoll M, de Ronde D, Perez LH, Sardon T, Vernos I and Schmit AC: The plant TPX2 protein regulates prospindle assembly before nuclear envelope breakdown. Plant Cell 20: 2783-2797, 2008.

5. Iyer J and Tsai MY: A novel role for TPX2 as a scaffold and co-activator protein of the chromosomal passenger complex. Cell Signal 24: 1677-1689, 2012.

6. Warner SL, Stephens BJ, Nwokenkwo S, Hostetter G, Sugeng A Hidalgo M, Trent JM, Han H and Von Hoff DD: Validation of TPX2 as a potential therapeutic target in pancreatic cancer cells. Clin Cancer Res 15: 6519-6528, 2009.

7. Scharer CD, Laycock N, Osunkoya AO, Logani S, McDonald JF, Benigno BB and Moreno CS: Aurora kinase inhibitors synergize with paclitaxel to induce apoptosis in ovarian cancer cells. J Transl Med 6: 79, 2008.

8. Shigeishi H, Ohta K, Hiraoka M, Fujimoto S, Minami M, Higashikawa K and Kamata N: Expression of TPX2 in salivary gland carcinomas. Oncol Rep 21: 341-344, 2009.

9. Wei P, Zhang N, Xu Y, Li X, Shi D, Wang Y, Li D and Cai S: TPX2 is a novel prognostic marker for the growth and metastasis of colon cancer. J Transl Med 11: 313, 2013.

10. Liu HC, Zhang Y, Wang XL, Qin WS, Liu YH, Zhang L and Zhu CL: Upregulation of the TPX2 gene is associated with enhanced tumor malignance of esophageal squamous cell carcinoma. Biomed Pharmacother 67: 751-755, 2013.

11. Lesuis SL, Maurin H, Borghgraef P, Lucassen PJ, Van Leuven F and Krugers HJ: Positive and negative early life experiences differentially modulate long term survival and amyloid protein levels in a mouse model of Alzheimer's disease. Oncotarget: Jun 1, 2016 (Epub ahead of print).

12. Mattsson N, Carrillo MC, Dean RA, Devous MD Sr, Nikolcheva T, Pesini P, Salter H, Potter WZ, Sperling RS, Bateman RJ, et al: Revolutionizing Alzheimer's disease and clinical trials through biomarkers. Alzheimers Dement Amst 1: 412-419, 2015.

13. Olsson B, Lautner R, Andreasson U, Öhrfelt A, Portelius E, Bjerke M, Hölttä M, Rosén C, Olsson C, Strobel G, et al: CSF and blood biomarkers for the diagnosis of Alzheimer's disease: a systematic review and meta-analysis. Lancet Neurol 15: 673-684, 2016.

14. Wolters FJ, Koudstaal PJ, Hofman A, van Duijn CM and Ikram MA: Serum apolipoprotein E is associated with long-term risk of Alzheimer's disease: The Rotterdam Study. Neurosci Lett 617: 139-142, 2016. 
15. Kamer AR, Fortea JO, Videla S, Mayoral A, Janal M, Carmona-Iragui M, Benejam B, Craig RG, Saxena D, Corby P, et al: Periodontal disease's contribution to Alzheimer's disease progression in Down syndrome. Alzheimers Dement Amst 2: 49-57, 2016.

16. Liu Q, Yang P, Tu K, Zhang H, Zheng X, Yao Y and Liu Q: TPX2 knockdown suppressed hepatocellular carcinoma cell invasion via inactivating AKT signaling and inhibiting MMP2 and MMP9 expression. Chin J Cancer Res 26: 410-417, 2014.

17. Huang Y, Guo W and Kan H: TPX2 is a prognostic marker and contributes to growth and metastasis of human hepatocellular carcinoma. Int J Mol Sci 15: 18148-18161, 2014.

18. Furukawa T, Kanai N, Shiwaku HO, Soga N, Uehara A and Horii A: AURKA is one of the downstream targets of MAPK1/ERK2 in pancreatic cancer. Oncogene 25: 4831-4839, 2006.
19. Koppara A, Wagner M, Lange C, Ernst A, Wiese B, König HH, Brettschneider C, Riedel-Heller S, Luppa M, Weyerer S, et al: Cognitive performance before and after the onset of subjective cognitive decline in old age. Alzheimers Dement Amst 1: 194-205, 2015.

20. Cukier HN, Kunkle BW, Vardarajan BN, Rolati S, Hamilton-Nelson KL, Kohli MA, Whitehead PL, Dombroski BA, Van Booven D, Lang R, et al; Alzheimer's Disease Genetics Consortium: ABCA7 frameshift deletion associated with Alzheimer disease in African Americans. Neurol Genet 2: e79. 2016.

21. Smithers BM, Li A, Kelly SL, Wilson MK, Chaturvedi A and Samoukovic K: Staging of non-small cell lung cancer and small cell lung cancer. Asian Pac J Surg Oncol 1: 125-140, 2015. 